\title{
The Specificity of Electrical Energy Storage Unit Application
}

\author{
Evgen Sokol, Volodymyr Zamaruiev, Bohdan Styslo, Mykola Makhonin \\ National Technical University "Kharkov Polytechnic Institute" (Ukraine) \\ Kharkov, Ukraine \\ bohdanstyslo@gmail.com
}

\section{INTRODUCTION}

The dissimilarity in the amounts of energy consumption during a day - is one of the main problems of Power engineering. A typical daily power consumption graph has its maximum and minimum areas that usually occur during daytime and nighttime respectively. As a result both excessive and insufficient power generation periods appear during a day.

Alongside the daily power consumption deviations, low durational peak power fluctuations occur in the grid. Alternating start and hold modes of additional power generators during the day noticeably increase both the wear out rate of generating equipment and fuel consumption (Every start of a generating hall operation requires additional fuel consumption). Apart from this, the technical ability of a generating object fast start is not always present [1].

An obvious way to balance the generated and consumed power is to include a battery energy storage system (BESS) into the power grid, which allows to decrease daily fluctuations of generated power and spread the energy accumulation and costumer supply phases of over time $[2,3]$.

Power fluctuation problem is present in the railway electricity network. During locomotive motion between two neighboring substations, a pulsed power consumption is observed. The consequence of this becomes increasing losses in the power supply line, unbalanced load on the feeding three-phase network, the maximum voltage drawdown.

The application of electrochemical energy storages in power system engineering allows to solve a wide range of problems such as: decreasing energy production costs by smoothing power generator load profile; increasing the life of grid equipment by lowering the peak load on substations; enabling the integration of solar and wind power generators into the existing power grid; improvement the quality of delivery of electrical energy; increasing the overall reliability of energy systems.

When choosing a special type of energy storage device for the BESS, it is necessary to take into consideration both technical and economic characteristics. The technical characteristics include charge-discharge cycle efficiency, charge time, overload capacity (output of pulsed currents), frequency characteristics (capacity to quickly transmit energy). Factors influencing the economic indicators include the unit cost of the battery, its lifetime.
As example, on the railway, BESS can be placed on a traction substations (TS) and preferably between them, or directly on the locomotive. The second variant is more preferable from energy considerations. When choosing the characteristics of the storage, it is necessary to take into consideration the area of applicability and tasks of the BESS. The final choice of the type of energy storage device is a compromise between technical and economical requirements.

The purpose of this paper is to consider the comparative characteristics of existing types of energy storage device, and the specificity of their usage as part of the BESS

\section{REQUIREMENTS FOR ELECTRIC ENERGY STORAGE DEVICES}

Despite the wide variety of electrical energy battery storages, the choice of accumulators for power engineering applications is restricted to units that meet specific requirements: high energy intensity; battery life (without maintenance); absence of a «memory effect»; availability of fast battery recharge modes; ability to operate under high current overloads; safety of usage; low cost.

Unfortunately none of the existing types of electrochemical batteries fully matches such requirements: despite being widely common, cheap, and able to operate under high current overloads, Lead-acid batteries fail to enable fast recharge modes, have low energy intensity, are ecologically hazardous and require specially equipped rooms. $\mathrm{NiCad}$ and $\mathrm{NiMH}$ batteries tolerate to current overload operation and have fast discharge/recharge rate, but have the memory effect (especially $\mathrm{NiCad}$ ) and a high self-discharge current. $\mathrm{LiCoO}_{2}$ and $\mathrm{LiMn}_{2} \mathrm{O}_{4}$ batteries show high energy performance, have long life, didn't have the memory effect, have low self-discharge rate, tolerate to high current overloads, capable of fast recharge/discharge, ecologically safe, have a sufficiently long battery life, but they are expensive and may become dangerous under abusive operating condition. $\mathrm{LiFePO}_{4}$ batteries posses all the positive features of other lithium battery types, while exceeding them in terms of battery life, but still feature the risk of explosion. The relatively recent appearance (in 2003) of this type of batteries on the market stands for their high price.

\section{FREQUENCY CHARACTERISTICS OF BATTERY}

Now many types of energy storages device are known, that differ from kind of storage energy and the design. Every type of energy storage has the own working mode, character 
constructional and energy parameters. The combinations of these characteristics determine rational exploitation field.

One of the essential energy characteristics of energy storage is the frequency range that possible effective energy exchange with storages (frequency characteristics). These characteristics are different for existing of electrical energy storage and usually do not overlap each over, which suggest the feasibility of hybrid storage of electrical energy (consist of storage with different frequency characteristics) for compensation pulsation of power consumption.

The Ragone plot [4] allows sorting different storage unit types by their time and energy characteristics. It is obvious if a specific battery cannot compensate high frequency power fluctuations, then a storage device of another type must compensate them. The graph analysis shows that electrochemical storage units of different types, capable of operating under various frequencies, are required to be plugged into the BESS structure to compensate grid power fluctuations in a sufficiently wide range of frequencies [2, 3, 5]. This decision allows, first of all, extending frequency range of storage, ensuring effective exchange of energy in each frequency domain. However, for design of the hybrid storage units methods that determine the installed capacity of each type of used storage units is need to develop.

The proposed method based on analysis statistical data of current and voltage at the site of contact network connection to its customers. Having the informational array of measurements consumed power of nonlinear load in the spectral composition of the currents can be identified frequency ranges for effective storage of various types (dF1 dF3 Fig. 1). Obviously, the spectrogram at very large data sample will adequately reflect the impact of load on the contact network in a controlled area.

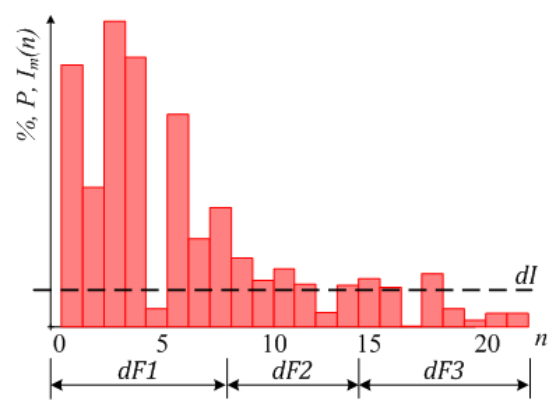

Fig. 1. Spectral composition of the currents.

Full capacity of hybrid storage is determined by the integral sum of samples consumed from the network current at a given frequency range. Assigned range of frequency diapasons in the spectrogram and the minimum significant amplitude of harmonics (dI) can be evaluate by the value of energy that may be received (transmitted) a specific type of storage unit. Thus, the percentage of capacities of each type of storage units is defined as the ratio of the integral sum of harmonic amplitudes throughout the frequency range to the integral sum of harmonic amplitudes in a given frequency range $\mathrm{dF}$. Connecting the hybrid storage device to the power supply network is available in two versions (Fig. 2, Fig. 3). In the first case, the operation of storage devices in the respective frequency range is provided by the difference in storage devices features.

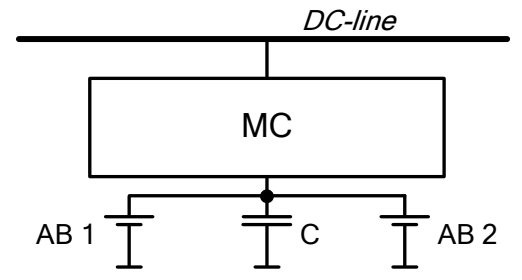

Fig. 2. Parallel connection different types of storage devices with common matching DC/DC converter: $\mathrm{MC}$ - Matching converter; AB1, AB2 accumulator batteries of different types; $\mathrm{C}$ - capacitor battery.

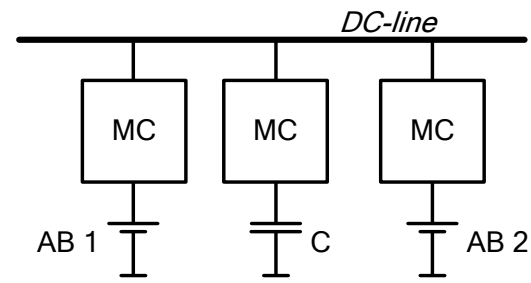

Fig. 3. Connection different types of storage devices with individual matching DC/DC converters.

This does not ensure accordance between currents and frequency characteristics of different types of batteries at low frequencies. Full compliance with the current consumed and the frequency characteristics of storage devices is provided in circuit with individual matching DC/DC converters.

\section{CONCLUSIONS}

It is shown that to increase efficiency it is necessary to use a hybrid BESS. Each type of energy storage device must be used in the frequency range of effective operation.

It is proposed to use LiFePO4 batteries in the BESS. The main positive features of this type of batteries are high specific energy intensity, high efficiency.

Battery operating parameters of AMP20M1HD-A such as the ratio between battery voltage and recharge time, frequency parameters of battery, variation of internal resistance of battery in discontinuous discharge mode were determined. It is determined that the battery makes it possible to form current pulses with a width 40 mos and over.

\section{REFERENCES}

[1] [Online] Available: http://www.branan.ru/uPower\%20review_5.pdf

[2] V. Zamaruiev, "Battery energy storage system for use in smart power systems" Energy saving, energetics, energy audit. Special edition, 2013. pp. $77-84$

[3] Damith B. "Single-Phase Grid-Connected LiFePO4 BatterySupercapacitor Hybrid Energy Storage System with Interleaved Boost Inverter" DOI 10.1109/TPEL.2014.2372774

[4] Ragone Chart Available: http://www.mpoweruk.com/performance.htm

[5] A. Kuperman and I. Aharon, "Battery-ultracapacitor hybrids for pulsed current loads: A review," Renewable and Sustainable Energy Reviews, vol. 15, pp. 981-992, 2011. 